\title{
ICI Reduction in Multicarrier Systems
}

\author{
Shaimaa EISayed \\ Ibrahim \\ Lecturer Assistant, \\ Dept. of EECT, \\ Modern Academy in Ma'adi, \\ Egypt
}

\author{
K. A. EIBarbary \\ Professor and Head of \\ Electric Engineering \\ Department, Suez Canal \\ University, Egypt
}

\author{
Ragab M. El-Sagheer \\ Lecturer at Dept. of Electrical \\ Communication, Al-Azhar \\ University in Cairo, Egypt
}

\begin{abstract}
Inter-Carrier Interference (ICI) is a major contribution to performance degradation in multicarrier systems. The main reason of the ICI is the Carrier Frequency Offset (CFO). Some researchers reduced the effect of this ICI by estimating the CFO value then compensating for it. The CFO estimation accuracy affects in turn the multicarrier system performance. The Repeated Prefix (RP) is a new technique that is recently proposed to reduce the ICI effect by estimating the CFO value without producing mathematical derivations. The drawback of this technique is its low performance at low guard interval values. This paper enhances the performance of this method at low guard interval values, giving the Maximum Likelihood Estimation (MLE) derivation for this process in addition to a derivation of the relative Cramer-Rao Lower Bound (CRLB). The proposed technique (RP-combined) reduces the effect of the residual ICI and can be used once in the transmitted frame with less data rate losses. Simulations show comparable results between both theoretical and simulated cases. Simulations also show the system performance with/without using channel estimation stage to show that, the channel estimator increases the immunity of the system performance to ICI for small offset values.
\end{abstract}

\section{Keywords}

Orthogonal frequency division multiplexing (OFDM), channel estimation, Repeated Prefix (RP), inter-carrier interference (ICI), carrier frequency offset (CFO), Maximum Likelihood Estimation (MLE), Cramer-Rao Lower Bound (CRLB).

\section{INTRODUCTION}

The demand of high data rate transmission through multipath channels makes the multicarrier communications are of great interest for researchers. Multicarrier systems like Digital Audio Broadcasting (DAB), Digital Video Broadcasting (DVB), Multicarrier Code Division Multiple Access (MCCDMA) and Long Term Term Evolution (LTE) are considered high data rates communication systems that use the popular multicarrier Orthogonal Frequency Division Multiplexing (OFDM) as a modulation technique. One drawback of the OFDM signal is its sensitivity to the InterCarrier Interference (ICI) problem which destroys the orthogonality between its subcarriers.

The main reason of the ICI is the Carrier Frequency Offset (CFO) that is generated as a result of Doppler Effect or the oscillators' instability used to generate the Radio Frequency (RF) carrier. The amount of this CFO $(\Delta f)$ is measured relative to the subcarrier spacing $\left(f_{\text {sub }}\right)$ in a normalized form $(\varepsilon)$, where $(\varepsilon)$ is the normalized CFO that may be fractional, integral or both values.
Researchers had proposed many techniques to reduce the effect of ICI in the multicarrier systems by estimating the CFO value first then compensating for it. Some of those researches used a special form of an OFDM symbol placed as a preamble in the beginning of the frame for the purpose of CFO estimation [1], [2], [3]. Where, a special form of OFDM symbol that is named Training Symbol (TS) is proposed, where it used a comb type frequency domain symbol construction to form a training of similar time-domain subblocks and hence the name TS. By the help of the repeated sub-blocks, the $\varepsilon$ value could then be estimated. In [1], a Best Linear Unbiased Estimator (BLUE) derivation is used for the $\varepsilon$ parameter estimation while in [3], a derivation of the Maximum Likelihood Estimation (MLE) of the $\varepsilon$ parameter is produced with an evaluation of the CFO estimator's variance using the Cramer-Rao lower bound (CRLB). The disadvantage of this TS method is its high computational complexity through the $\mathrm{CFO}$ estimation process in addition to reducing the transmitted data rate as it adds an OFDM symbol as a redundancy at every transmitted frame.

Other researchers used the inherent construction of the conventional Cyclic Prefix (CP) based OFDM symbol to estimate the CFO value. Such blind techniques use reduced computational complexity and save the transmitted data rate as it is not need to send any extra redundancy [4]. In [4], [5].a MLE for $\varepsilon$ parameter estimation and CRLB is derived, but unfortunately, this CP-based method can only reduce the effect of the ICI for low CFO values, where $0 \leq|\varepsilon|_{C P} \leq 0.5$ which is a limited range. Another new technique was proposed in [6], [7] where a new OFDM symbol construction is used to extend the CFO estimation range by four times at least compared to the CP-based technique. This was performed by the help of sending another guard interval redundancy samples to the transmitted OFDM symbol. This new technique, named Repeated Prefix (RP), can be used in both CP-based OFDM systems and Zero Padded (ZP) OFDM systems. In [7], the technique is first presented while in [6], a comparison between the RP-based and CP-based techniques is produced showing the validity of the RP-symbol to be used in ZP-based OFDM systems. The RP-technique showed comparable results with the offset free case in terms of bit error rate curves for high $N$ and $N_{g} / N$ values but this performance was degraded for low $N$ and $N_{g} / N$, where $N$ is the number of orthogonal subcarriers of the OFDM system and $N_{g}$ is the number of samples in the guard interval portion of an OFDM symbol.

In [6], [7], the RP-technique is presented as a new ICI reduction technique by estimating the $\mathrm{CFO}$ value then compensating for its effect, evaluating the OFDM system performance using MATLAB simulations with bit error rate 
curves while the mean square error of the estimator did not considered. In this paper a derivation of the $\operatorname{MSE}(\varepsilon)$ using the CRLB is introduced and compared the theoretical results with the simulated results. A MLE derivation for the parameter $\varepsilon$ is also presented in this paper.

In this paper, a method is proposed also to reduce the ICI effect for low $N$ and $N_{g} / N$ values using the RP technique for the purpose of enhancing its accuracy in those cases. Mathematical derivations for the MLE of $\varepsilon$ estimation and its relative CRLB for variance evaluation are also presented in this paper to determine the parameters affecting the CFO estimation process. Unlike [6], [7], in which the RP symbol is used as an alternative to the conventional OFDM symbol, resulting in decreasing the transmitted bandwidth efficiency, it is also proposed in this paper to use the RP symbol as a preamble once at the beginning of the transmitted frame and thus the transmitted data rate is saved even compared to the other preamble techniques like TS technique. Simulations show comparable results in terms of Mean Square Error (MSE) between theoretical and simulated cases. Simulations show also the OFDM system performance with/without using a channel estimation stage to show the system immunity to ICI effect in the presence of the channel estimation stage for low $\mathrm{CFO}$ values.

The rest of the paper is organized as follows: in Section 2, an overview of OFDM system as a multicarrier technique is introduced, showing the orthogonality concept. The ICI problem is presented in Section 3 while the MLE and CRLB derivations are introduced in Section 4. The proposed technique with its relative MLE and CRLB is presented in Section 5. In Section 6, simulations and results are found. Finally, conclusions are introduced in Section7.

\section{OFDM AS A MULTICARRIER TECHNIQUE}

OFDM is considered a multicarrier technique as it converts the serial data bits to transmitted symbols $\{X(k)\}$ that are either M-PSK or M-QAM mapped then dividing them into blocks of length $N$ that are used as input to an Inverse Fast Fourier Transform (IFFT) stage to modulate them onto Northogonal subcarriers, constructing a time-domain samples $x(n)$, given by

$$
x(n)=\frac{1}{N} \sum_{k=0}^{N-1} X(k) e^{\frac{j 2 \pi n k}{N}}, n, k=0: N-1
$$

Where, $k$ is a subcarrier index used at the transmitter side and each frequency $(2 \pi k / N)$ represents a subcarrier which is orthogonal to the other subcarriers.

\subsection{The Orthogonality Concept}

Two signals are orthogonal if their inner product is zero as,

$$
\sum \cos \left(\frac{2 \pi k n}{N}\right) * \cos \left(\frac{2 \pi k m}{N}\right)=0, n \neq m
$$

In frequency domain, this means that, the spectrum of each subcarrier has a null at the centre of the spectrum of other subcarriers. This is achieved in the OFDM system by the help of the IFFT stage as shown in Equation 1.

To prevent the transmitted OFDM symbol from Inter Symbol Interference (ISI), a guard interval with length $N_{g}$ is then added before transmission. This guard interval may contain a copy of last $N_{g}$ samples of the IFFT output $x(n)$, where the transmitted OFDM symbol $s(n)$ is then takes the form,

$$
s(n)= \begin{cases}x(n+N) & \text { if } \quad-N_{g} \leq n<0 \\ x(n) & \text { if } 0 \leq n \leq N-1\end{cases}
$$

The guard interval in this case is named Cyclic Prefix (CP). CP preserves the orthogonality of the OFDM signal, permitting simple equalization process at the receiver side.

\subsection{Multipath Propagation}

In case of multipath channels, channel state information (CSI) is required at the receiver side to equalize for its effect. In this case, the transmission of known pilot symbols $X_{P}(k)$ may be added to the transmitted signal at the transmitter side for the purpose of multipath channel estimation. After converting from parallel to serial, the signal is up-converted on a Radio Frequency (RF) carrier signal $f_{c}$ and then transmitted through a multipath channel exposed to fading and Additive White Gaussian Noise (AWGN) effects.

In multipath channels, the signal is received from $v$-paths. The operation can be modeled as linear convolution operation [8] as the multipath channel can be modeled as a finite impulse response filter. This linear convolution operation is simply a multiplication in frequency domain by the help of Discrete Time Fourier Transform (DTFT). But the continuity of the signal in frequency makes the computations of the multiplication operation to be impossible on digital computers [8]. Hence, DTFT cannot be used to perform linear convolution operation and discrete signals are required to be used instead of continuous signals.

Therefore, FFT which has the property of circular convolution is used instead to obtain such a linear filtering operation. So, if the output of the linear convolution operation was equivalent to the circular convolution operation, the equalization process at receiver side can then be simply obtained using one tap frequency domain equalizer. If the orthogonality concept is maintained, this operation can be achieved in the OFDM system by the help of the CP part [6]

\subsection{OFDM Receiver}

The received signal is first down converted from the $f_{c}$ using a local oscillator then converted from serial to parallel before removing the $\mathrm{CP}$ part and then converted to the frequency domain using a FFT stage. The frequency domain received pilots $\left\{Y_{P}(l)\right\}$ are extracted to estimate the multipath channel's coefficients $\widehat{H}(l)$. In this paper, the DFT-based Least Square (LS) channel estimation [9] is used, where,

$$
\widehat{H}_{D F T-L S}(l)=Y_{P}(l) / X_{P}(k)
$$

These channel estimated coefficients $\widehat{H}_{D F T-L S}(l)$ are then used by a frequency domain equalizer to equalize for the channel's effects and get the equalized frequency domain data symbols $\{\hat{X}(l)\}$ which is then de-mapped to obtain the received data serial bits.

\section{INTERCARRIER INTERFERENCE PROBLEM}

In the presence of mobility, a Doppler frequency is generated depending on the velocity (vel) and the $f_{c}$ used, where a maximum Doppler frequency $f_{D}$ is presented as,

$$
f_{D}= \pm \frac{v e l * f_{c}}{c}
$$

Where $c$ is the velocity of light in the air. Taking the instability of the local oscillators used to generate the RF carrier frequency into considerations $\left(f_{\text {osc }}\right)$ that is $\pm 10 \mathrm{PPM}$ (Parts Per Million) of the carrier frequency [10], a total $\mathrm{CFO}(\Delta f)$ is generated, where, $\Delta f=f_{o s c}+f_{D}$. This total 
$\mathrm{CFO}, \Delta f$ causes the receiver to sample away from the centers of the subcarriers as shown in Figure 1, destroying the orthogonality and resulting in ICI between the subcarriers. This leads to system performance degradation.

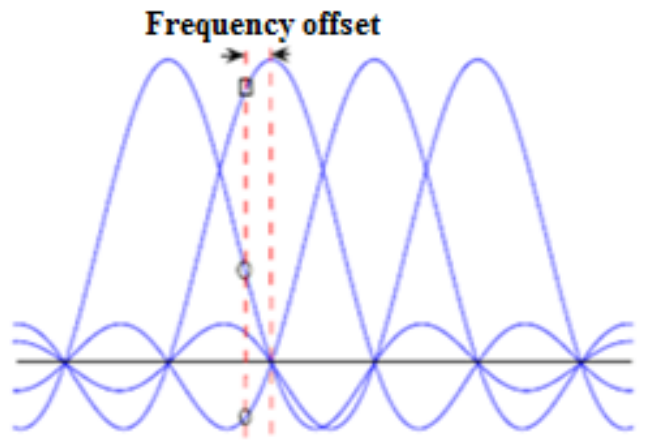

Fig 1: ICI due to CFO in the OFDM spectrum

The normalized CFO is then the sum of $\varepsilon_{D}=f_{D} / f_{\text {sub }}$ and $\varepsilon_{\text {osc }}=f_{\text {osc }} / f_{\text {sub }}$, where $\varepsilon_{D}$ takes small fractional values [11] while $\varepsilon_{o s c}$ takes larger values (both integer and fractional values). However, the interference coefficients $S(l-k)$ is expressed as [6],

$$
S(k-l)=e^{j \pi[k+\varepsilon-l]\left(1-\frac{1}{N}\right)} * \frac{\sin \pi(k+\varepsilon-l)}{N \sin \left(\frac{\pi}{N}(k+\varepsilon-l)\right)}
$$

As the channel estimation operation depends on the orthogonality principle, a CFO of $(\varepsilon)$ affects its operation and hence leads to performance degradation. So, in this case, it is highly required to reduce the ICI effect by estimating the CFO value then compensating for it before using the channel estimation and equalization stages.

\section{RP TECHNIQUE WITH MLE AND CRLB DERIVATIONS}

In [7], a new ICI reduction technique, named Repeated Prefix (RP), was proposed to reduce the ICI effect over large CFO values (multiple of subcarrier spacing). This technique used a new OFDM symbol construction which is named RP symbol. this symbol is constructed by modifying the conventional OFDM symbol construction. In this RP symbol, a copy of the first $N_{g}{ }^{R P}$ samples of the IFFT output is inserted at the front of the transmitted OFDM signal (and hence the name Repeated Prefix (RP)) before adding the CP part. For the purpose of saving the transmitted data rate and keeping reliable CFO estimation accuracy, the value of this $N_{g}{ }^{R P}$ was chosen to be the same value as $N_{g}$. In this paper, the symbols $N_{g}{ }^{R P}$ and $N_{g}$ is used interchangeably. In the RP symbol a $2 N_{g}$ redundancy samples are added to the transmitted symbol while just $N_{g}$ redundancy samples are added to the conventional transmitted CP-based OFDM symbol. The RP symbol is then takes the form,

$$
s(n)=\left\{\begin{array}{cl}
x\left(n+N+N_{g}\right) & \text { if }-2 N_{g} \leq n<-N_{g} \\
x\left(n+N_{g}\right) & \text { if }-N_{g} \leq n<0 \\
x(n) & \text { if } 0 \leq n \leq N-1
\end{array}\right.
$$

This RP symbol construction is shown in Figure 2, Where the redundancy in the $\mathcal{L}$ part is used for CFO estimation purpose and the redundancy in $\mathcal{L}_{C P}$ part is used to convert the linear convolution operation to the circular convolution operation to use one tap frequency domain equalization stage as used in the conventional OFDM symbol case, keeping the same receiver complexity [7], [6].

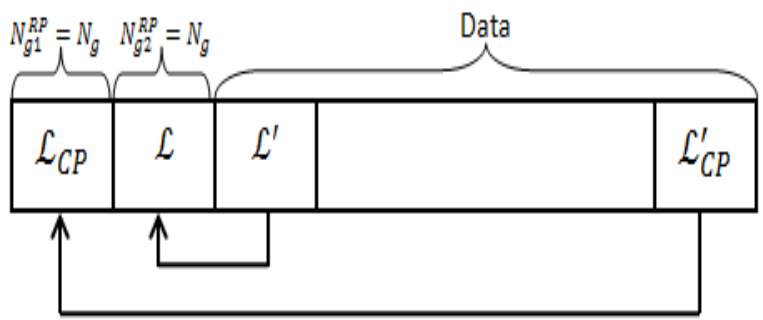

Fig 2: RP symbol structure used in CP-based OFDM system

When the transmitted OFDM symbol $s(n)$ is affected by $\varepsilon$, assuming that $\varepsilon$ is distributed equally over positive and negative sides around zero, an assumption that is valid for narrowband systems [12], the received signal will have a complex multiplicative factor with an increasing phase value in time (a property of Fourier transform) and then takes the form:

$$
r(n)=s(n) e^{\frac{j 2 \pi \varepsilon n}{N}}
$$

Considering transmission of one RP-symbol with $\left(N+N_{g}\right)$ length (ignoring the samples in the $\left(\mathcal{L}_{\mathrm{CP}}\right)$ part) where the samples in the extended part $(\mathcal{L})$ and their copies separated by $N_{g}$ samples in $\left(\mathcal{L}^{\prime}\right)$ are pair wise correlated while the remaining samples $r(n), n \notin \mathcal{L} \cup \mathcal{L}^{\prime}$ are uncorrelated. Concerning the observation period of $2 N_{g}$ samples long $[\mathcal{L} \cup$ $\left.\mathcal{L}^{\prime}\right]$, these samples in the observation period is pair wise correlated i.e:

$\forall n \in \mathcal{L}: E\left\{r(k) r^{*}(n+m)\right\}=\left\{\begin{array}{cc}\sigma_{s}^{2}+\sigma_{n}^{2} & m=0 \\ \sigma_{s}^{2} e^{-j 2 \pi \varepsilon \frac{N_{g}}{N}} & m=N_{g} \\ 0 & \text { Otherwise }\end{array}\right.$

Where, $E\{\cdot\}$ is the expected value which is a sum of $N_{g}$ consecutive correlations between pairs of samples spaced $N_{g}$ apart, $\sigma_{s}^{2}$ and $\sigma_{n}^{2}$ are the sample's power and noise variance, respectively.

Depending on the correlation properties between $\mathcal{L}$ and $\mathcal{L}$ parts, a $\operatorname{MLE}(\varepsilon)$ and its relative CRLB derivations are presented in this paper.

\subsection{Maximum Likelihood Estimation (MLE) derivation for the RP technique}

The likelihood of the parameter, $\varepsilon$, given the outcomes $r$, is equal to the probability of those observed outcomes $r$ given the parameter value $\varepsilon$. For many applications, the natural logarithm of the likelihood function, called the log-likelihood, is more convenient to work with.

The method of maximum likelihood estimation selects the value of the parameter $(\varepsilon)$ that maximizes the likelihood function by taking the logarithm of the probability density function of the observed data first then takes its derivative with respect to the parameter $(\varepsilon)$.

Using the MLE method, the $\log$-likelihood function for $\varepsilon$ is the logarithm of the joint probability density function $f(r / \varepsilon)$ of the observed samples in $r$, given the normalized carrier frequency offset $\varepsilon$.

Using the correlation properties of the observation $r$, the $\log$ likelihood function $\Lambda(\varepsilon)$ can be written as,

$$
\Lambda(\varepsilon)=\log _{e}(f(r / \varepsilon))
$$


Where

$$
\begin{gathered}
f(r / \varepsilon)=\prod_{n} f(r(n)) \\
=\prod_{n \in \mathcal{L}} \frac{f\left(r(n), r\left(n+N_{g}\right)\right)}{f(r(n)) f\left(r\left(n+N_{g}\right)\right)} \prod_{n} f(r(n))
\end{gathered}
$$

Where $f(r(n))$ and $f\left(r\left(n+N_{g}\right)\right)$ are the marginal univariate complex normal distribution [5], given by,

$$
\begin{gathered}
f(r(n))=\frac{1}{\pi\left(\sigma_{s}^{2}+\sigma_{n}^{2}\right)} e^{-\frac{|r(n)|^{2}}{\left(\sigma_{s}^{2}+\sigma_{n}^{2}\right)}} \\
, f\left(r\left(n+N_{g}\right)\right)=\frac{1}{\pi\left(\sigma_{s}^{2}+\sigma_{n}^{2}\right)} e^{-\frac{\left|r\left(n+N_{g}\right)\right|^{2}}{\left(\sigma_{s}^{2}+\sigma_{n}^{2}\right)}}
\end{gathered}
$$

Which have a zero mean and variance of $\left(\sigma_{s}^{2}+\sigma_{n}^{2}\right)$. Since the maximum likelihood (ML) estimation of $\varepsilon$ is the argument that maximizing the $f(r / \varepsilon)$ and the product $\prod_{n} f(r(n))$ in Equation 11 is rotationally invariant over all $(n)$ and hence independent of $\varepsilon$, this product factor can be omitted and the $\log$ likelihood function $\Lambda(\varepsilon)$ can then be written as,

$$
\begin{gathered}
\Lambda(\varepsilon)=\log _{e}(f(r / \varepsilon)) \\
=\ln \left(\prod_{n \in \mathcal{L}} \frac{f\left(r(n), r\left(n+N_{g}\right)\right)}{f(r(n)) f\left(r\left(n+N_{g}\right)\right)}\right)
\end{gathered}
$$

While $f\left(r(n), r\left(n+N_{g}\right)\right)$ is the joint bivariate complex normal distribution, given by,

$$
\begin{aligned}
f\left(r(n), r\left(n+N_{g}\right)\right) & =f(r(n)) f\left(r\left(n+N_{g}\right)\right) \\
& =\frac{1}{\pi^{2}|\Sigma|} e^{-\left[\boldsymbol{R}^{H} \Sigma^{-1} \boldsymbol{R}\right]}
\end{aligned}
$$

Where, $\boldsymbol{R}$ is a vector containing the number of variables which is two $\left(r(n) \& r\left(n+N_{g}\right)\right)$ in the bivariate case and $\boldsymbol{R}^{\boldsymbol{H}}$ is the Hermitian transpose (complex transpose) of $\boldsymbol{R}$ and hence,

$$
\boldsymbol{R}^{\boldsymbol{H}}=\left[\mathrm{r}^{*}(\mathrm{n}) \mathrm{r}^{*}\left(\mathrm{n}+\mathrm{N}_{\mathrm{g}}\right)\right]
$$

While $|\Sigma|$ is the determinant of the covariance matrix $\Sigma$ that given by,

$$
\Sigma=\left[\begin{array}{ll}
\sigma_{11} & \sigma_{12} \\
\sigma_{21} & \sigma_{22}
\end{array}\right]
$$

Where, $\sigma_{i j}$ is the covariance between $i$ and $j$, using the correlation properties in Equation 16, the covariance matrix can be written as,

$$
\begin{gathered}
\Sigma=\left[\begin{array}{cc}
\sigma_{s}^{2}+\sigma_{n}^{2} & \sigma_{n, n+N_{g}} \\
\sigma_{n+N_{g}, n} & \sigma_{s}^{2}+\sigma_{n}^{2}
\end{array}\right] \\
\Sigma=\left[\begin{array}{cc}
\sigma_{s}^{2}+\sigma_{n}^{2} & \left|\rho_{n}\right|\left(\sigma_{s}^{2}+\sigma_{n}^{2}\right) e^{-j 2 \pi \varepsilon \frac{N_{g}}{N}} \\
\left|\rho_{n}\right|\left(\sigma_{s}^{2}+\sigma_{n}^{2}\right) e^{j 2 \pi \varepsilon \frac{N_{g}}{N}} & \sigma_{s}^{2}+\sigma_{n}^{2}
\end{array}\right]
\end{gathered}
$$

Where $\left|\rho_{n}\right|$ is the magnitude of the correlation coefficient $\left(\rho_{n}\right), \forall n \in \mathcal{L}$, computed as,

$$
\rho_{n}=\frac{E\left\{r(n) r^{*}\left(n+N_{g}\right)\right\}}{\sqrt{\sigma_{s}^{2}+\sigma_{n}^{2}} \sqrt{\sigma_{s}^{2}+\sigma_{n}^{2}}}=\left|\rho_{n}\right| e^{-j 2 \pi \varepsilon \frac{N_{g}}{N}}
$$

Where the correlation coefficient $\rho_{n}$ is a measure of linearity between two random variables and is measured as:

$$
\rho_{n}=\frac{\operatorname{cov}\left(r(n), r\left(n+N_{g}\right)\right)}{\operatorname{std}(r(n)) \operatorname{std}\left(r\left(n+N_{g}\right)\right)}=\frac{\sigma_{n, n+N_{g}}}{\left(\sigma_{s}^{2}+\sigma_{n}^{2}\right)}
$$

And hence, $\quad|\Sigma|=\left(\sigma_{s}^{2}+\sigma_{n}^{2}\right)^{2}\left(1-\left|\rho_{n}\right|^{2}\right)$

Substituting Equations 15, 21 into Equation 14, and after some manipulations, Equation 14 can then rewritten as,

$$
f\left(r(n), r\left(n+N_{g}\right)\right)=\frac{\exp \left(-\frac{A}{\left(\sigma_{s}^{2}+\sigma_{n}^{2}\right)\left(1-\left|\rho_{n}\right|^{2}\right)}\right)}{\pi^{2}\left(\sigma_{s}^{2}+\sigma_{n}^{2}\right)^{2}\left(1-\left|\rho_{n}\right|^{2}\right)}
$$

Where, $A=|r(n)|^{2}-2\left|\rho_{n}\right| \operatorname{Re}\left\{e^{j 2 \pi \varepsilon \frac{N g}{N}} r(n) r^{*}\left(n+N_{g}\right)\right\}+$ $\left|r\left(n+N_{g}\right)\right|^{2}$. Using Equation`12, the log likelihood function can be rewritten as,

$$
\Lambda(\varepsilon)=\sum_{n=-N_{g}}^{-1}-\ln \left(1-\left|\rho_{n}\right|^{2}\right)+\frac{B}{\left(\sigma_{s}^{2}+\sigma_{n}^{2}\right)\left(1-\left|\rho_{n}\right|^{2}\right)}
$$

Where, $B=2\left|\rho_{n}\right| \operatorname{Re}\left\{e^{j 2 \pi \varepsilon \frac{N_{g}}{N}} \gamma(n)\right\}-\left|\rho_{n}\right|^{2}\left(|r(n)|^{2}+\right.$ $\left.\left|r\left(n+N_{g}\right)\right|^{2}\right)$ and $\gamma(n)=r(n) r^{*}\left(n+N_{g}\right)$. Let,

$$
\begin{aligned}
\operatorname{Re}\left\{e^{j 2 \pi \varepsilon \frac{N g}{N}} \gamma(n)\right\} & =\operatorname{Re}\left\{e^{j 2 \pi \varepsilon \frac{N g}{N}}|\gamma(n)| e^{j \angle \gamma(n)}\right\} \\
& =|\gamma(n)| \cos \left(2 \pi \varepsilon \frac{N_{g}}{N}+\angle \gamma(n)\right)
\end{aligned}
$$

The ML estimate of $\varepsilon$, is the argument that maximizes the $\log$ likelihood function in Equation 23 that is occurred when the $\operatorname{term}\left(2 \pi \varepsilon \frac{N_{g}}{N}+\angle \gamma(n)\right)=0$.

$$
\begin{gathered}
\therefore \quad-2 \pi \hat{\varepsilon} \frac{N_{g}}{N}=\angle \gamma(n) \\
\therefore \quad \hat{\varepsilon}=\frac{-N}{2 \pi N_{g}} * \arg \left[\sum_{n=-N_{g}}^{-1} r(n) r^{*}\left(n+N_{g}\right)\right]
\end{gathered}
$$

Or equivalently,

$$
\hat{\varepsilon}=\frac{N}{2 \pi N_{g}} * \arg \left[\sum_{n=-N_{g}}^{-1} r^{*}(n) r\left(n+N_{g}\right)\right]
$$

If $(P)$ OFDM symbols are used, $\hat{\varepsilon}$ can be averaged as,

$$
\hat{\varepsilon}_{P}=\frac{N}{2 \pi N_{g}} * \arg \left[\frac{1}{P} \sum_{p=0}^{P-1} \sum_{n=-N_{g}}^{-1} r_{p}^{*}(n) r_{p}\left(n+N_{g}\right)\right]
$$

Since $\mathrm{P}$ is positive integer, division by $\mathrm{P}$ does not change the sign and hence the value of the angle, then it can be omitted from the above equation, as,

$$
\hat{\varepsilon}_{P}=\frac{N}{2 \pi N_{g}} * \arg \left[\sum_{p=0}^{P-1} \sum_{n=-N_{g}}^{-1} r_{p}^{*}(n) r_{p}\left(n+N_{g}\right)\right]
$$

In a multipath channel, the number of these analogous samples (in $\mathcal{L}, \mathcal{L}^{\prime}$ parts) is decreased as the channel length $(v)$ increase. The CFO can then be estimated from the product of the extended and the corresponding parts of the received RPOFDM symbol by taking $\left\{-N_{g}+v-1 \leq n \leq-1\right\}$ in Equations 27 and 29 [6], [7].

The CFO estimation range in the RP-technique can then evaluated as the argument operation is limited to $[-\pi: \pi]$, then the CFO estimation range in the RP-technique will be:

$$
\left|\hat{\varepsilon}_{R P}\right|=\frac{N \pi}{2 \pi N_{g}}=\frac{N}{2 N_{g}}
$$

This means that, the RP-technique can reduce the ICI effect for $\mathrm{CFO}$ values limited by Equation 30. The CFO estimation range is therefore inversely proportional to the $\left(N_{g} / N\right)$ ratio. However, for maximum $\left(N_{g} / N=1 / 4\right)$ ratio used in the OFDM systems, the maximum normalized CFO estimation range is $\left|\hat{\varepsilon}_{R P}\right| \cong 2$ which is four times than that can be obtained from the CP-based techniques [4], [5], [6] while 
for $\left(N_{g} / N=1 / 32\right)$, the maximum normalized CFO estimation range is $\left|\hat{\varepsilon}_{R P}\right| \cong 16$. Since it is considered a wide range (the estimated $\mathrm{CFO}$ value can be multiple of subcarrier spacing), the estimated normalized offset value of Equations 27 and 29 is then named $\left|\hat{\varepsilon}_{R P \text {-wide }}\right|$ in this paper.

The variance of an estimator is an important function associated with any estimator that is useful of how well the estimator is doing. In the next section, a CRLB derivation is introduced to set the variance of the RP technique, showing the parameters affecting the system performance.

\subsection{Cramer-Rao Lower Bound (CRLB) derivation for the RP technique}

The Cramer Rao Lower Bound (CRLB) expresses a lower bound on the variance of estimators of a deterministic parameter which is the normalized carrier frequency offset $(\varepsilon)$ in this case. It is widely used as a benchmark to evaluate the performance of an estimation algorithm [13].

The variance of any unbiased estimator is bounded by the reciprocal of the Fisher information. Where, the Fisher information is a way of measuring the amount of information that an observable random variable carries about an unknown parameter and is measured by using the expectation of the second derivative of the $\log$ likelihood function with respect to this parameter as $-\mathrm{E}\left\{\partial^{2} \Lambda_{\mathrm{P}}(\varepsilon) / \partial \varepsilon^{2}\right\}[13]$.

This section produces the CRLB and variance of the RPproposed technique to show the corresponding Mean Square Error $(\operatorname{MSE}(\varepsilon))$ due to the $\mathrm{CFO}$ estimation process.

For $(P)$ OFDM received symbols, let,

$$
\Phi(n)=\frac{1}{P} \sum_{p=0}^{P-1} \phi_{p}(n)
$$

Where

$$
\begin{array}{r}
\phi_{p}(n)=\frac{1}{2}\left(\left|r_{p}(n)\right|^{2}+\left|r_{p}\left(n+N_{g}\right)\right|^{2}\right) \\
\Gamma(n)=\frac{1}{P} \sum_{\mathrm{p}=0}^{\mathrm{P}-1} \operatorname{Re}\left\{\mathrm{e}^{\mathrm{j} 2 \pi \varepsilon \frac{\mathrm{Ng}}{\mathrm{N}}} \gamma(\mathrm{n})\right\}
\end{array}
$$

The log-likelihood function in Equation 23 can then written for $(P)$ OFDM symbols as,

$$
\begin{aligned}
& \Lambda_{P}(\varepsilon)= \\
& P \sum_{n=-N_{g}}^{-1}-\ln \left(1-\left|\rho_{n}\right|^{2}\right)+\frac{2|\rho|}{\left(\sigma_{s}^{2}+\sigma_{n}^{2}\right)\left(1-\left|\rho_{n}\right|^{2}\right)}(\Gamma(n)- \\
& \left.\frac{\left|\rho_{n}\right|}{2} \Phi(n)\right)
\end{aligned}
$$

Combining Equation 24, Equation 33 and Equation 34 then taking the second derivative of Equation 34 with respect to $(\varepsilon)$, after some manipulations,

$$
\begin{aligned}
& \therefore \frac{\partial^{2} \Lambda_{P}(\varepsilon)}{\partial \varepsilon^{2}}= \\
& \sum_{n=-N_{g}}^{-1} \frac{-8 \pi^{2} P\left(\frac{N_{g}}{N}\right)^{2}\left|\rho_{n}\right|}{\left(\sigma_{s}^{2}+\sigma_{n}^{2}\right)\left(1-\left|\rho_{n}\right|^{2}\right)}\left(\frac{1}{P} \sum_{p=0}^{P-1} \operatorname{Re}\left\{e^{j 2 \pi \varepsilon \frac{N_{g}}{N}} \gamma(n)\right\}\right)
\end{aligned}
$$

The Fisher information can then written as,

$$
\begin{gathered}
-\mathrm{E}\left\{\frac{\partial^{2} \Lambda_{\mathrm{P}}(\varepsilon)}{\partial \varepsilon^{2}}\right\}=8 \pi^{2} \mathrm{P}\left(\frac{\mathrm{N}_{\mathrm{g}}}{\mathrm{N}}\right)^{2} * \\
\sum_{\mathrm{n}=-\mathrm{N}_{\mathrm{g}}}^{-1} \frac{\left|\rho_{\mathrm{n}}\right|}{\left(\sigma_{\mathrm{s}}^{2}+\sigma_{\mathrm{n}}^{2}\right)\left(1-\left|\rho_{\mathrm{n}}\right|^{2}\right)}\left(\frac{1}{\mathrm{P}} \sum_{\mathrm{p}=0}^{\mathrm{P}-1} \operatorname{Re}\left\{\mathrm{e}^{\mathrm{j} 2 \pi \varepsilon \frac{\mathrm{Ng}}{\mathrm{N}}} \mathrm{E}\{\gamma(\mathrm{n})\}\right\}\right)
\end{gathered}
$$

Where, $E\{\gamma(n)\}$ is the expectation of $\gamma(n)$. As $\gamma(n)=$ $r(n) r^{*}\left(n+N_{g}\right)$ and using the relations in Equation 9, Equation 36 can be rewritten as,

$$
-E\left\{\frac{\partial^{2} \Lambda_{P}(\varepsilon)}{\partial \varepsilon^{2}}\right\}=8 \pi^{2} P\left(\frac{N_{g}}{N}\right)^{2} \sum_{n=-N_{g}}^{-1} \frac{\left|\rho_{n}\right|}{\left(1-\left|\rho_{n}\right|^{2}\right)} \frac{\sigma_{s}^{2}}{\left(\sigma_{s}^{2}+\sigma_{n}^{2}\right)}
$$

From Equation 20, as the magnitude of the correlation coefficient $\left(\left|\rho_{n}\right|\right)$ can be written as $\left(\left|\rho_{n}\right|=\frac{\sigma_{s}^{2}}{\left(\sigma_{s}^{2}+\sigma_{n}^{2}\right)}\right)$, the Fisher information can rewritten as,

$$
-E\left\{\frac{\partial^{2} \Lambda_{P}(\varepsilon)}{\partial \varepsilon^{2}}\right\}=8 \pi^{2} P\left(\frac{N_{g}}{N}\right)^{2} \sum_{n=-N_{g}}^{-1} \frac{\left|\rho_{n}\right|^{2}}{\left(1-\left|\rho_{n}\right|^{2}\right)}
$$

So, the Cramer lower bound (CRLB) can be computed as,

$$
\begin{gathered}
C R L B=\frac{1}{\text { Fisher information }}=\left[-E\left\{\frac{\partial^{2} \Lambda_{P}(\varepsilon)}{\partial \varepsilon^{2}}\right\}\right]^{-1} \\
C R L B=\frac{1}{8 \pi^{2} P\left(\frac{N g}{N}\right)^{2} \sum_{n=-N}^{-1} \frac{\left|\rho_{n}\right|^{2}}{\left(1-\left|\rho_{n}\right|^{2}\right)}}
\end{gathered}
$$

Hence, the variance of the RP technique can then be calculated as,

$$
\begin{gathered}
E\left[\left(\hat{\varepsilon}_{P}-\varepsilon\right)^{2}\right] \geq \frac{1}{8 \pi^{2} P\left(\frac{N g}{N}\right)^{2} \sum_{n=-N g}^{-1} \frac{\left|\rho_{n}\right|^{2}}{\left(1-\left|\rho_{n}\right|^{2}\right)}} \\
\left|\rho_{n}\right|=\frac{\sigma_{s}^{2}}{\left(\sigma_{S}^{2}+\sigma_{n}^{2}\right)}=\frac{S N R}{S N R+1}
\end{gathered}
$$

Then, the variance is lower bounded by the CRLB as,

$$
E\left[\left(\hat{\varepsilon}_{P}-\varepsilon\right)^{2}\right] \geq\left(\frac{1}{8 \pi^{2} P\left(\frac{N g}{N}\right)^{2}\left(N_{g}\right)}\right)\left(\frac{2 S N R+1}{S N R^{2}}\right)
$$

From Equation 43, it is clear that, the parameters affecting the variance or the $\operatorname{MSE}(\varepsilon)$ of the RP technique are the number of received OFDM symbols that used in the averaging of the estimation process $(\mathrm{P})$, the number of samples contained in the extended $\left(\mathrm{N}_{\mathrm{g}}\right)$ part, the ratio of $\left(\mathrm{N}_{\mathrm{g}} / \mathrm{N}\right)$, and the value of the signal to noise ratio (SNR).

Note, Equation 43 can be used in the case of a multipath channel also as the received signal power is $\sigma_{s}^{2}$ in case of AWGN of Equation 42 is replaced with $\sigma_{s}^{2} \sum_{v}|h(v)|^{2}$, where $|h(v)|^{2}$ is the gain power of the received $v$-path and using the index $n=-N_{g}+v-1:-1$ instead of $n=-N_{g}:-1$. As the guard interval length in the OFDM system is chosen to be at least twice the channel impulse response length (for practical considerations) [14]. In worst case the summation term in case of the multipath channel will be limited by $N_{g} / 2$.

\section{PROPOSED RP-COMBINED TECHNIQUE}

As seen the accuracy in terms of $\operatorname{MSE}\left(\varepsilon_{R P \text {-wide }}\right)$ of the $\mathrm{CFO}$ estimation process in the RP scheme determined with Equation 43 is affected by the guard interval length, where at low guard interval lengths, the corresponding $\operatorname{MSE}\left(\varepsilon_{R P \text {-wide }}\right)$ increases which is considered a problem that may degrade the system performance at low guard interval length. So, it is suggested to use the RP technique proposed in [6], [7] in the case of using high $N$ and $N_{g}$ lengths as it still can cover wide CFO estimation range $\left(\hat{\varepsilon}=\varepsilon_{R P-\text { wide }}>1\right)$. In this case the RP technique is named RP-wide.

However, this paper proposes to enhance the RP-wide performance at this case where, the MSE of the CFO estimation process can be enhanced if a second stage is used to estimate the residual value $\left(\varepsilon_{\text {residual }}\right)$. As the RP-symbol contains another repeated samples in the first and last $N_{g}^{R P}$ parts of $\mathcal{L}_{\mathrm{CP}}, \mathcal{L}_{\mathrm{CP}}^{\prime}$, the residual $\varepsilon$ can be then estimated. However, this two stage process can be used in the case of using low guard interval lengths, maintaining the advantage of 
high $\mathrm{CFO}$ estimation range. In this case, the RP-technique is named RP-combined as the estimation process is a result of a combination of two stages $\left(\varepsilon_{R P-\text { wide }}, \varepsilon_{R P-N a r r o w}\right)$ that results in $\left(\varepsilon_{R P}\right.$-combined $)$. The RP-combined steps are shown in Figure 3 .

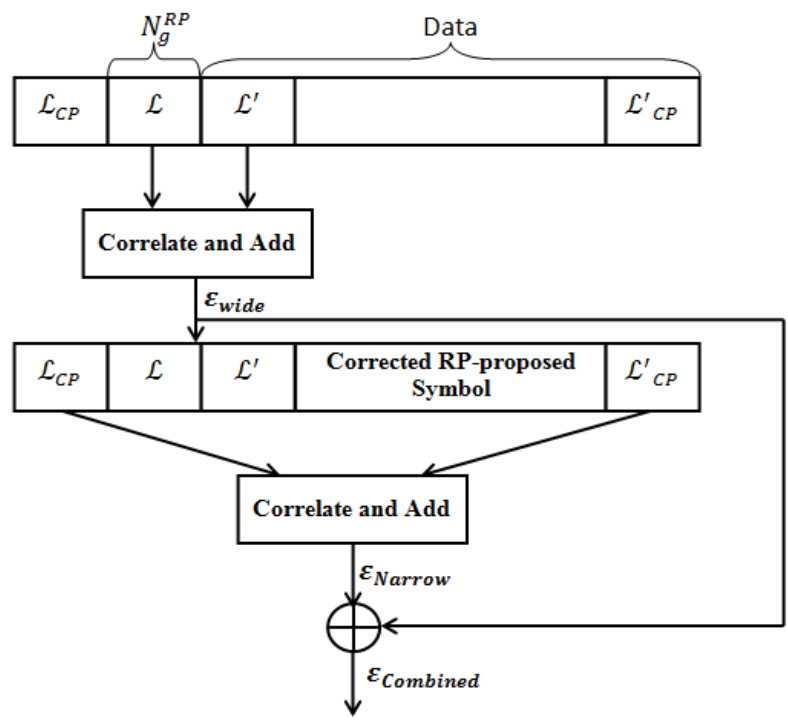

Fig 3: CFO estimation procedure used in the proposed RP-combined technique

Following the same steps of the MLE derivations discussed, considering the $\mathcal{L}_{\mathrm{CP}}$ and $\mathcal{L}_{\mathrm{CP}}^{\prime}$ samples that are spaced $(N+$ $\left.N_{g}^{R P}\right)$ samples apart, the residual CFO can be estimated using:

$\hat{\varepsilon}_{R P-N a r r o w}=\frac{N}{2 \pi\left(N+N_{g}^{R P}\right)} * \arg \left[\sum_{n=0}^{N_{g}-1} r^{*}(n) r(n+N+\right.$ $\left.\left.N_{g}^{R P}\right)\right]$

So, the CFO estimation range in this stage is limited to,

$$
\left|\hat{\varepsilon}_{R P-N a r r o w}\right|=\frac{N \pi}{2 \pi\left(N+N_{g}^{R P}\right)}=\frac{N}{2\left(N+N_{g}^{R P}\right)}
$$

This results in narrowing the estimation range to be $\hat{\varepsilon}_{R P-\text { Narrow }}=\left[(-N:+N) / 2\left(N+N_{g}^{R P}\right)\right]$ in the second stage. The corresponding CRLB is found to be:

$$
\begin{aligned}
& E\left[\left(\hat{\varepsilon}_{R P-\text { Narrow }}-\varepsilon_{\text {residual }}\right)^{2}\right] \geq \\
& \left(\frac{1}{8 \pi^{2}\left(\frac{N+N_{g}^{R P}}{N}\right)^{2}\left(N_{g}^{R P}\right)}\right)\left(\frac{2 S N R+1}{S N R^{2}}\right)
\end{aligned}
$$

Comparing the accuracy of the second stage in Equation 46 with that of the first stage in Equation 43, the variance found to be reduced by $\left(\frac{N+N_{g}^{R P}}{N_{g}^{R P}}\right)^{2}=\left(1+\frac{N}{N_{g}^{R P}}\right)^{2}$ times. So, increasing the accuracy of the estimator can be met for low guard interval lengths by either averaging over $(P)$ OFDM symbols or using the RP-combined method with just one RPsymbol.

In [6], [7], the RP-symbol is used as an alternative to the conventional OFDM symbol, using the same receiver design as in the conventional OFDM system with a simple replacement operation of the received samples to convert the linear convolution operation to circular convolution operation as in CP-based OFDM systems. This has been met with the advantage of using low computational complexity (depending on the $N_{g}$ length) and over wide CFO estimation range. As the RP-symbol used as an alternative to the conventional OFDM symbol, the system was able to update the estimated CFO value many times during the frame and used $(P)$ OFDM symbols to enhance the system accuracy for low $\mathrm{N}$ and $N_{g}$ lengths. These advantages were achieved at the expense of lowering the transmitted data rate, where the RP-symbol needs to send an extra $2 N_{g}$ redundancy samples to the transmitted symbol instead of sending just $N_{g}$ samples in the conventional OFDM symbol.

Using the proposed RP-combined technique adds the advantage of estimating the CFO value with higher estimation accuracy using just one OFDM symbol instead of averaging over $(P)$ OFDM symbols. In this way, the RP-symbol can be used as a preamble once at the beginning of the frame of the conventional OFDM system. This saves the transmitted data rate compared to the RP technique of [6], [7] and even compared to other preamble methods as TS technique of [1], [2], [3]. Where, in the RP-combined, an extra redundancy of $2 N_{g}$ samples are required to be transmitted once per frame while in TS technique a redundancy of a complete OFDM symbol of length $\left(N+N_{g}\right)$ is sent once per frame. In the simulations of this paper, the RP-symbol is used as a preamble in the beginning of a frame consists of conventional CP-based OFDM symbol.

\section{SIMULATIONS AND RESULTS 6.1 ICI Effect on OFDM System Performance}

The immunity of the OFDM to ICI caused by CFO is shown in Figure 4 with/without using DFT-based LS channel estimation stage. As seen, in the offset free case, using the channel estimation stage adds a small error to the OFDM system for low $E b / N O$ values. However, in the case of small CFO values of $\varepsilon=0.05$, using the channel estimation stage makes the system more immune to the ICI effect compared to the "without channel estimation" case, where it saves about $11 \mathrm{~dB}$ of $E b / N O$ at $\mathrm{P}(\mathrm{e})=0.03$. Unfortunately, at higher $\mathrm{CFO}$ values as $\varepsilon=1.2$, both cases have a poor bit error rate of about 0.5 . So, it is highly required to use an ICI reduction technique in this case or even for $\varepsilon=0.05$ case to compensate for this performance degradation.

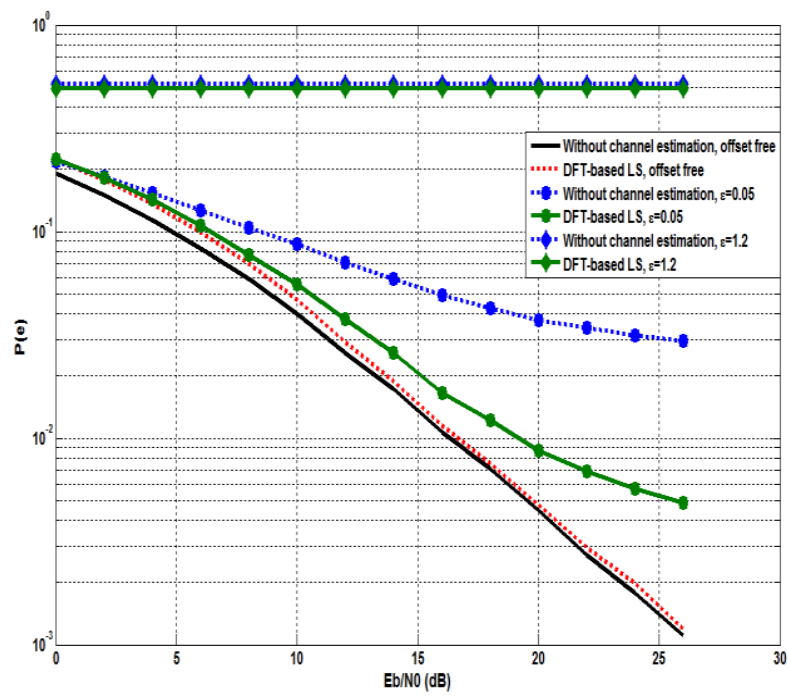

Fig 4: OFDM performance in the presence of CFO with/without using channel estimation stage, 16QAM 


\subsection{MSE Considerations}

This section measures the MSE for the RP technique and compares it with the theoretical CRLB derived in Equation 43 , for different SNR values, where $\varepsilon=1.2$.

As the CRLB is affected by the used number of orthogonal subcarriers $(\mathrm{N})$ and the number of RP-symbols used in the average process (P), Figure 5 compares the theoretical CRLB with the MSE got from simulations for different N, P values.

As shown, the simulated MSE shows comparable results with the derived theoretical CRLB. It is also shown that, the MSE is inversely proportional to $\mathrm{P}, \mathrm{N}$ values, where, at $\mathrm{SNR}=10 \mathrm{~dB}$, the MSE for $\mathrm{N}=64, \mathrm{P}=1$ takes the highest value of about $2.5^{*} 10^{-3}$ while for $\mathrm{N}=64, \mathrm{P}=16$, the MSE is decreased to be about $2 * 10^{-4}$ and it is the lowest for $\mathrm{N}=8192, \mathrm{P}=1$ case to be about $2 * 10^{-5}$

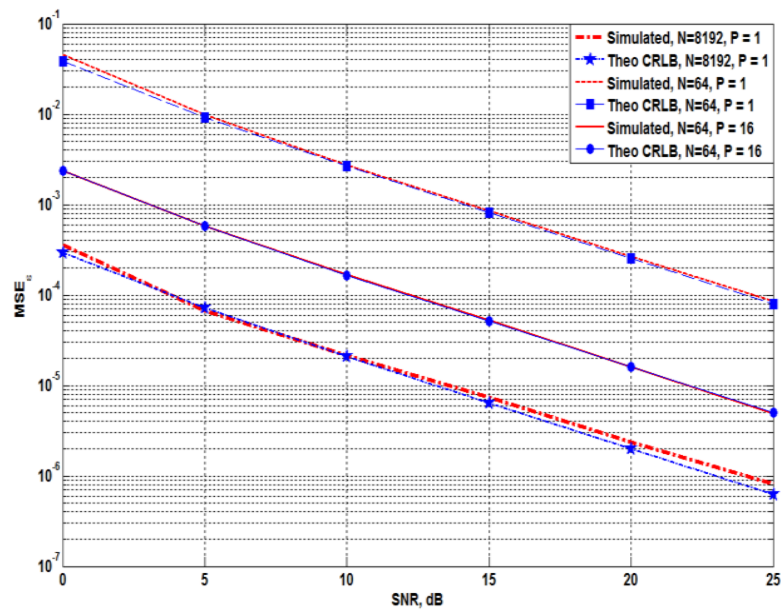

Fig 5: Theoretical CRLB with simulations comparisons, for different $\mathbf{N}, \mathbf{P}$ values

As the CRLB depends also on the cyclic prefix length, Figure 6 shows the MSE for different $\left(N_{g} / N\right)$ ratios. As shown, the derived theoretical CRLB shows comparable results with the simulated curves. it is also shown that, the MSE is inversely proportional to the cyclic prefix length, where, at $\mathrm{SNR}=10 \mathrm{~dB}$, the MSE is about $5^{*} 10^{-3}$ in case of $\left(N_{g} / N\right)=1 / 32$ while it is decreased in case of $\left(N_{g} / N\right)=1 / 16$ to be about $7 * 10^{-4}$ while it is the lowest in case of $\left(N_{g} / N\right)=1 / 8$ to be about $10^{-4}$.

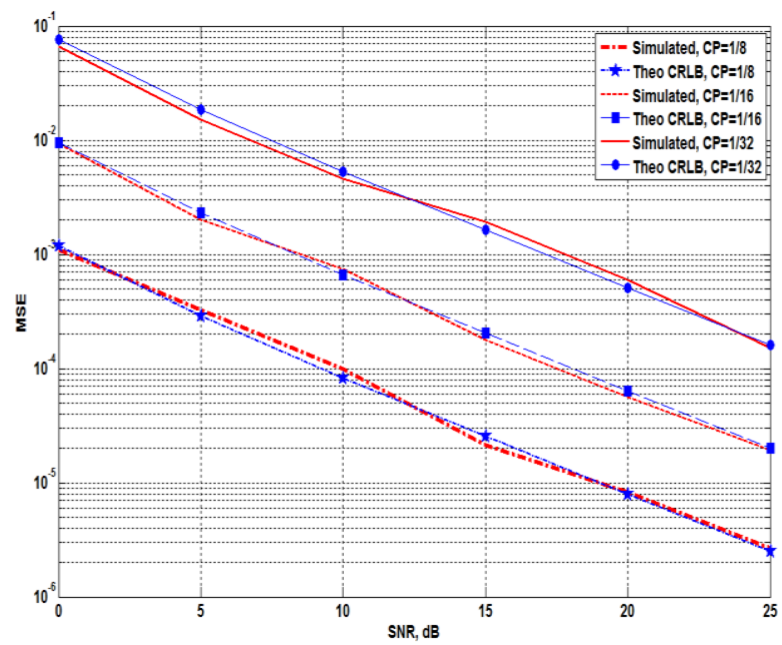

Fig 6: Theoretical CRLB with simulations comparisons, for different cyclic prefix lengths

\subsection{CFO Estimation Range Considerations}

This section shows the MSE for different $\varepsilon$ values to show the $\mathrm{CFO}$ estimation range for the RP-wide technique. Figure 7 shows this for different $P, N$ values. As shown, the CFO estimation range (which gives approximately the same MSE value) is about $|\varepsilon| \leq 2$, this is because the used $\left(N_{g} / N\right)=1 / 4$.

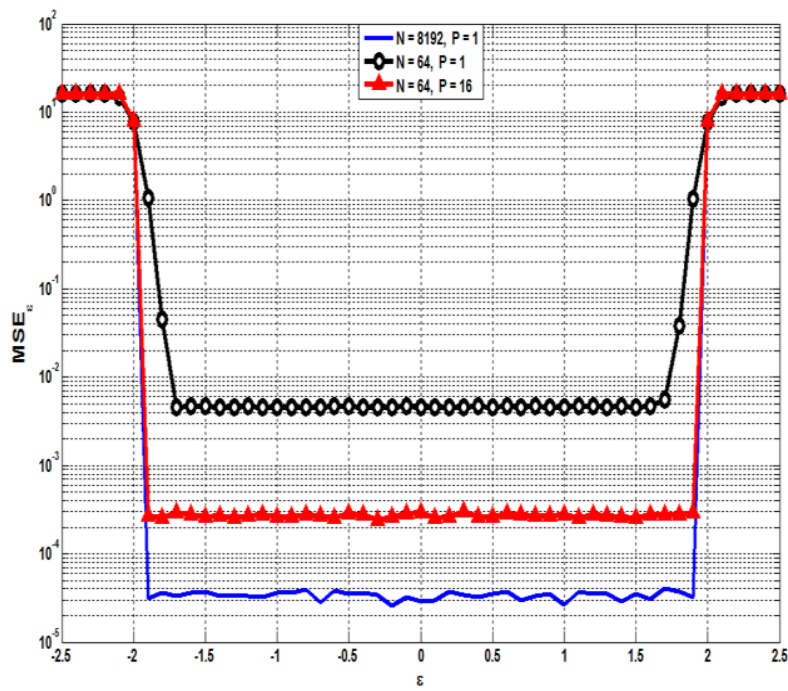

Fig 7: CFO estimation range in terms of MSE for different $\mathbf{N}, \mathbf{P}$ values

Figure 8 shows this relation for different $\left(N_{g} / N\right)$ values. It is shown that, the CFO estimation range is inversely proportional to the $\left(N_{g} / N\right)$ ratio, where at $\left(N_{g} / N\right)=1 / 8$, the CFO estimation range is 4 while it is 8 for $\left(N_{g} / N\right)=1 / 16$ and it is 16 for $\left(N_{g} / N\right)=1 / 32$.

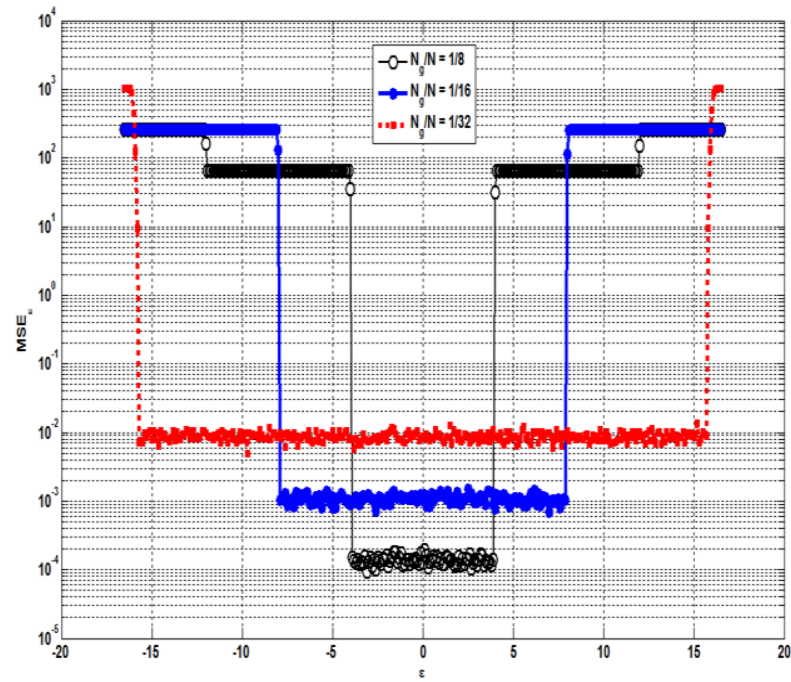

Fig 8: CFO estimation range in terms of MSE for different $\left(N_{g} / N\right)$ values

\subsection{Comparisons with the Proposed RP- combined method}

This section shows the performance comparisons between RP-wide and proposed RP-combined method in terms of MSE and bit error rate. The bit error rate curves uses the DFT-based LS channel estimation stage for both 16QAM and 64QAM mapped symbols. 
Figure 9 uses $N=8192,\left(N_{g} / N\right)=1 / 8$. As shown, the MSE is enhanced in case of the proposed RP-combined case relative to the RP-wide case where at SNR=10dB, the MSE is about $3 * 10^{-4}$ in case of RP-wide while it is about $4 * 10^{-6}$ in the case of the proposed RP-combined method.

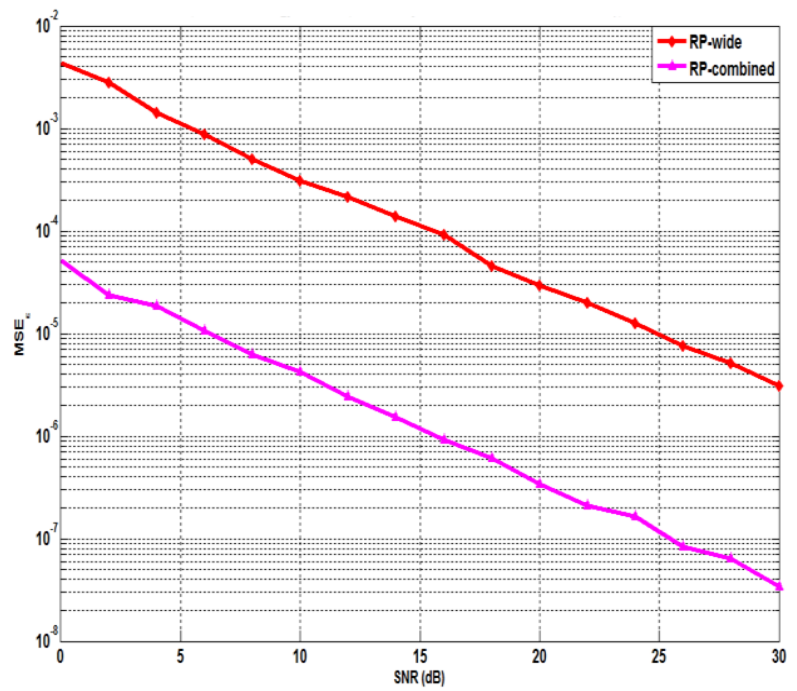

Fig 9: MSE comparison between RP-wide and proposed RP-combined ICI reduction methods, $\mathbf{N}=\mathbf{8 1 9 2}$, $\left(N_{g} / N\right)=1 / 8, \varepsilon=1.2$

The measure of the system performance is the bit error rate rather than the MSE while the MSE is used to show the comparisons for different systems. Figure 10 shows the bit error rate comparisons using 16QAM, 64QAM for both the $\mathrm{RP}$-wide and proposed RP-combined schemes in the presence of $\varepsilon=1.2$, using $\mathrm{N}=8192,\left(N_{g} / N\right)=1 / 8$. As shown, although the MSE of the RP-wide is larger than that of the proposed RP-combined method, both show comparable results with the offset free case. So, in this case, it is preferred to use the RPwide technique because it needs just one CFO estimation stage.

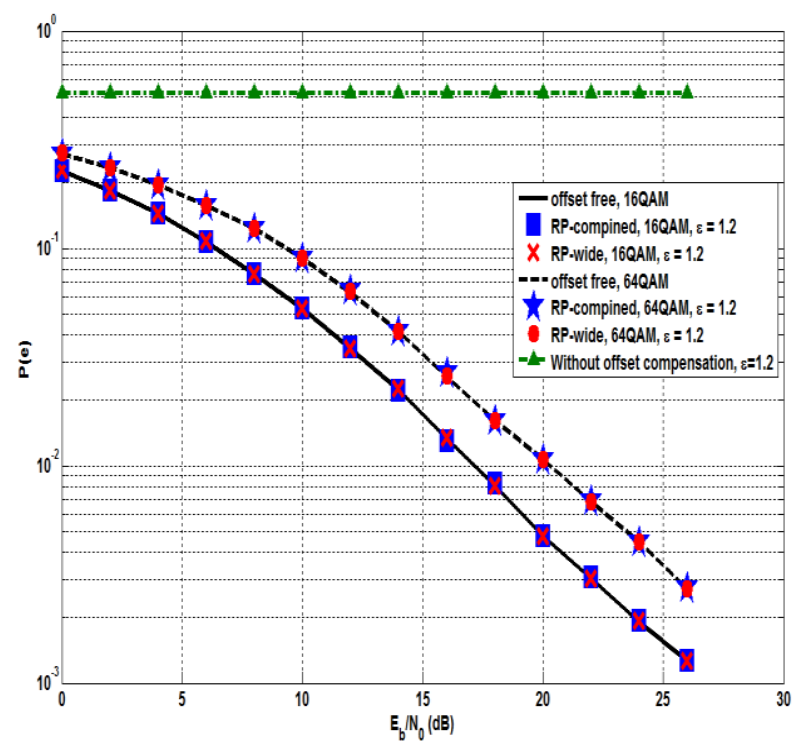

Fig 10: Bit error rate comparison between RP-wide and proposed RP-combined method in the presence of $\varepsilon=1.2$, $\mathrm{N}=8192,\left(N_{g} / N\right)=1 / 8$
In the case of using lower $\left(N_{g} / N\right)=1 / 32$, as shown in Figure 11, the RP-wide curve shows a slight degradation for $E b / N O \leq 18 \mathrm{~dB}$, where at bit error rate of $\mathrm{P}(\mathrm{e})=0.06$, there is a loss in $E b / N O$ by about $1 \mathrm{~dB}$ compared to the offset free case. While the proposed RP-combined curve still has comparable results with the offset free curve.

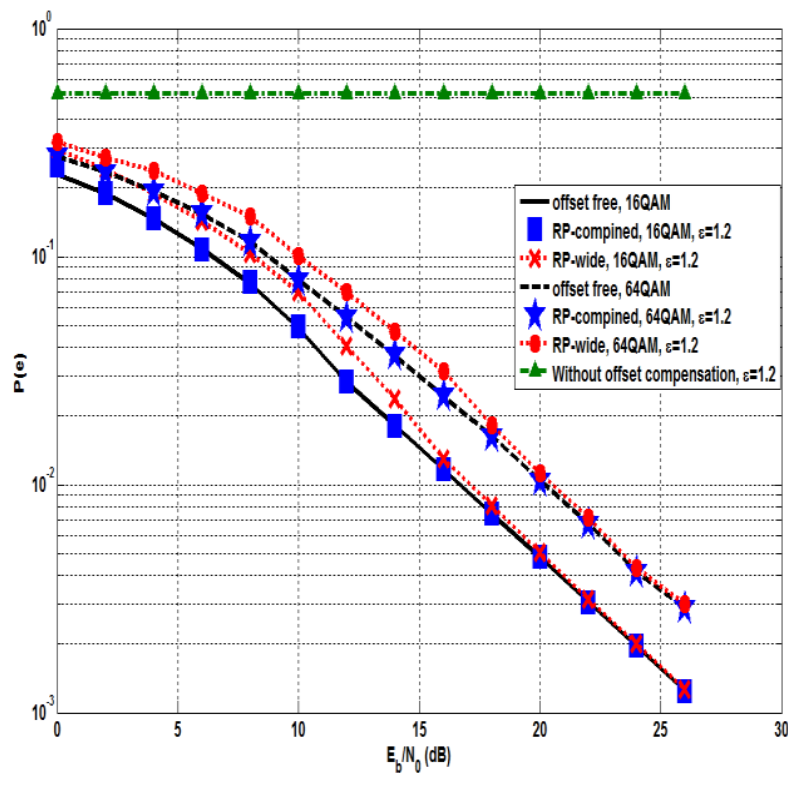

Fig 11: Bit error rate comparison between RP-wide and proposed RP-combined method in the presence of $\varepsilon=1.2$, $\mathrm{N}=8192,\left(N_{g} / N\right)=1 / 32$

\section{CONCLUSIONS}

The MSE of any estimator is a measure of its performance. In this paper a maximum likelihood derivation of the RP technique is presented. The CRLB derivation is also presented in this paper showing the parameters affecting its performance of the RP technique as an ICI reduction technique in multicarrier systems. It is found that, the MSE of the RP technique is inversely proportional to the used number of orthogonal subcarriers $(\mathrm{N})$, number of the RP-symbols used in the average process $(\mathrm{P})$, the $\left(N_{g} / N\right)$ ratio and signal to noise ratio (SNR).

In terms of bit error rate, the RP technique showed comparable results with the offset free curve at high $\left(N_{g} / N\right)$ ratio of $1 / 8$, using $\mathrm{N}=8192$ while a slight degradation of the system performance appeared when using lower $\left(N_{g} / N\right)$ ratio of $1 / 32$ where at $\mathrm{P}(\mathrm{e})=0.06$, the corresponding $E b / N O$ loss was about $1 \mathrm{~dB}$ compared with the offset free curve.

This paper proposed also the RP-combined method to enhance the RP performance at low $\left(N_{g} / N\right)$ ratio. The MLE and CRLB of the RP-combined method is also presented in this paper. The MSE of the RP-combined method outperformed that of the RP technique and the bit error rate of the proposed RP-combined curve showed comparable results with the offset free curve at $\left(N_{g} / N\right)=1 / 32$.

The paper proposed also to use the RP-symbol once at the beginning of the frame as a preamble for ICI reduction purpose. In this way, the transmitted data rate is enhanced compared with the case of using the RP symbol as an alternative to the conventional OFDM symbol. 


\section{REFERENCES}

[1] M. M. a. U. Mengali, "An Improved Frequency Offset Estimator for OFDM Applications," in IEEE, Communication Theory Mini-Conference, Vancouver, BC, 1999.

[2] Y.-H. Y. J.-H. P. a. Y.-S. C. Hyoung-Kyu Song, "Frequency-Offset Synchronization and Channel Estimation for OFDM-Based Transmission," IEEE Communications Letters, vol. 4, no. 3, pp. 95-97, March 2000 .

[3] J. L. Q. Z. a. H.-L. L. Ji-Woong Choi, "Joint ML Estimation of Frame Timing and Carrier Frequency Offset for OFDM Systems Employing Time-Domain Repeated Preamble," IEEE Transactions on Wireless Communications, vol. 9, no. 1, pp. 311-317, Jan. 2010.

[4] M. S. a. P. O. B. Jan-Jaap van de Beek, "ML Estimation of Time and Frequency Offset in OFDM Systems," IEEE Transactions on Signal Processing, vol. 45, no. 7, pp. 1800-1805, July 1997.

[5] W.-L. Chin, "ML Estimation of Timing and Frequency Offsets using Distinctive Correlation Characteristics of OFDM signals over Dispersive Fading Channels," IEEE Transactions of Vehicular Technology, vol. 60, no. 2, pp. 444-456, Feb. 2011.

[6] K. E. a. R. M. E.-S. Shaimaa ElSayed Ibrahim, "CFO Mitigation in OFDM Systems with a Comparative Analysis to CP-Based Estimator Technique," in IEEE 32nd National Radio Science Conference (NRSC 2015), 6th of October City, 2015
[7] K. E. a. R. M. E.-S. Shaimaa ElSayed Ibrahim, "Maximizing CFO Estimation Range using a New OFDM Symbol Structure," International Journal of Computer Applications (0975-8887), vol. 88, no. 4, pp. 5-13, Feb. 2014.

[8] S. Sharma, Digital Signal Processing: with Matlab Programs, fifth edition, 2009.

[9] J. K. W. Y. Y. a. C. G. K. Yong Soo Cho, MIMO-OFDM wireless communications with MATLAB, (Asia) Pte Ltd: John Wiley \& Sons, 2010.

[10] Q. W. a. M. Rupp, "Analytical Link Performance Evaluation of LTE Downlink with Carrier Frequency Offset," in IEEE Conference Record of the Forty Fifth Asilomar Signals, Systems and Computers (ASILOMAR), Pacific Grove, CA, 2011.

[11] F. H. a. G. Matz, Wireless Communications over Rapidly Time-Varying Channels, Academic Press: Elsevier, 2011.

[12] G. a. R. M. Jeffrey G. Andrews, Fundamentals of WiMAX Understanding Broadband Wireless Networking, Pearson Education, Inc, 2007.

[13] Steven M. Kay, Fundamentals of Statistical Signal Processing: Estimation Theory, Prentice Hall, 1993.

[14] S. Ahmadi, Mobile WiMax: A System Approach to Understanding IEEE $802.16 \mathrm{~m}$ Radio Access Technology, ISBN: 978-0-12-374964-2: Elsevier, 2011. 